the decisions. The former techniques were more commonly observed in the second study whereas the latter strategies were more typically used in Study 1. Systematic attempts to categorize these behavioral interactions may prove to be fruitful in terms of generating hypotheses.

Adolescent males were used in this research since they would seem to be a prime group for study: They appear prone to "pecking orders" in gang activities and, in terms of crime statistics, they are the most aggressive age group in the population. The measure itself, however, was developed along lines suggested by Mann (1959), who reports that the number of task contributions by a member of a group correlates highly with other (pencil-and-paper) measures of dominance for that member. In line with Mann's reports, the strategy used in the present research might be workable with almost any group in the population. Children might be asked to decide among sets of paired toys. Females might be asked to decide between sets of paired pictures of attractive males or between sets of paired photos of attractive clothes.

One modification of the method would be to have a common stooge compete with the Ss. The stooge could be trained to respond in a standard, prescribed manner to each $S$ and his behavior rated by independent, unseen observers.

Another modification would be to use pairs of photos where both girls were, say, blond and blue-eyed, but one girl has been, in prior work, rated far prettier than the other. The numbers under the girls' photos would be reversed for the two members of the dyad, so that the picture of the homely girl would be Photo 1 for the LD S, but Photo 2 for the HD S. If, under these conditions, the HD S dominated the team's choices, one could feel confident about the dominance relationship between the two Ss.

\section{REFERENCES}

ALTROCCHI, J. Dominance as a factor in interpersonsl choice and perception. Journal of Abnormal \& Social Psycholozy, 1959, 59, 303-308.

HSIAO, S., \& SCHREIBER, S. C. Social dominance and motivational variables in rats. Psychonomic Science, 1968, 10, 117-118.

KRECH, D., \& CRUTCHFIELD, R. S. Theory and problems of social psychology. New York: McGraw-Hill, 1948.

MANN, R. D. A review of the relationships between personality and performance in small groups. Psychological Bulletin, 1959, 56, 241-270.

UYENO, E. T., \& WHITE, M. Social isolation and dominance behavior. Journal of Comparative \& Physiological Psy chology, 1967, 63, 157-159.

WLSON, W. J. Adaptation to the dominance tube. Psychonomic Science, $1968,10,119-120$.

\section{NOTE}

1. We wish to thank the officials and boys of the Lookout Mountain School for Boys of Golden, Colorado, for their cooperation in this research.

\title{
A note on methods of indexing associative relatedness'
}

PAUL E. JOHNSON ${ }^{2}$ and RAYMOND O. COLLIER, JR., UNIVERSITY OF MINNESOTA, Minneapolis, Minn. 55455

$A$ method of indexing response relations among stimulus words on a word association test is presented utilizing the concept of mathematical sets. Two coefficients of associative relatedness are proposed and some relationships between these and a number of other coefficients are given.

Methods for indexing relationships among the stimulus words on a word-association test have been presented by Deese (1962), Garskoff and Houston (1963), and Marshall and Cofer (1963). One group of methods defines the relatedness between two stimulus words as the ratio of the number of common responses to a basal measure that is a function of the total number of responses to the stimulus words. The specification of the number of common responses (the commonality) between two stimulus words is fairly well agreed upon by different investigators, except perhaps for the issue of representational or implicit responses (Deese, 1962, 1965). However, the basal measure between two stimulus words has been written in a number of ways. In the case of discrete association (each $S$ gives only one response to each stimulus word), Deese (1965) defines this basal quantity as the geometric mean of the total number of responses to the two stimulus words, while Marshall and Cofer (1963) define the basal measure as the sum of the number of responses for two stimulus words. For continued association (each S gives at least one response to each stimulus word) the total number of responses often varies among stimulus words and among Ss whereas the basal measure for two stimulus words for a given $\mathrm{S}$ can be written in terms of the number of responses in either the larger or the smaller of the two distributions. In the case of continued association, the commonality between two response distributions can also be formulated with a weighting factor so as to take account of the order in which responses are given (Garskoff \& Houston, 1963).

Coefficients of relatedness have been constructed, in the case of discrete association, for the purpose of indexing the response commonality among more than two stimulus words. Here, however, the counting rule used for determining commonality is different from the rule used for only two stimulus words, and also varies from coefficient to coefficient. Two such coefficients that have been proposed for two or more stimulus words are the index of total association (ITA) and the index of concept cohesiveness (ICC) (Marshall \& Cofer, 1963).

Methods of indexing associative relatedness can be conveniently classified according to whether they are based upon discrete or continued word association, and, jointly, according to whether they are formulated for two or for more than two stimulus words. None of the coefficients referred to previously were developed explicitly to deal with the situations in all categories in this 2 by 2 classification scheme. It is the purpose of this paper (a) to suggest a basis for defining a class of coefficients that is appropriate for discrete and continuous association and for two or more stimulus words, and (b) to develop explicitly coefficients for the case of discrete association and two or more stimulus words.

We consider the responses to a single stimulus word to be elements of a mathematical set. Responses to two or more 
stimulus words can then be viewed as elements of two or more sets and can thus be characterized as being members of either the intersection or the union of two or more sets. We then define the intersection set as one containing the elements (responses) that are common to all the stimulus words being compared, and the overlap set as one that contains the elements common to two or more of these stimulus words.

An intersection coefficient is formulated as the ratio of the number of words in the intersection set of responses to two or more stimulus words, to the number of words in the union of these sets of responses. Similarly, an overlap coefficient is formulated as the ratio of the number of words in the union of all two-way intersection sets of responses to the stimulus words being compared, to the number of words in the union of these sets of responses (the denominator or basal measure is the same for both coefficients).

For two stimulus words the intersection and overlap coefficients $\left(S_{I}\right.$ and $\left.S_{0}\right)$ are identical and are written as

$$
S_{1}=S_{0}=\frac{n_{12}}{n_{1}+n_{2}-n_{12}}
$$

where $n_{1}$ is the total number of words in the set of responses $\left(A_{1}\right)$ to one of the stimulus words, $n_{2}$ is the number of words in the set of responses $\left(A_{2}\right)$ to a second stimulus word, and $n_{12}$ is the number of words which $A_{1}$ and $A_{2}$ have in common (i.e., the number of words in the intersection of Sets $A_{1}$ and $A_{2}$ ). For discrete association the relationship between this formulation and the coefficient of mutual frequency (MF) or mutual relatedness (MR) (Marshall \& Cofer, 1963) and the coefficient proposed by Deese $(1962,1965)$ (D) can be obtained by writing these latter coefficients as

$$
M R=M F=\frac{n_{12}}{n_{1}+n_{2}} ; \quad D=\frac{n_{12}}{\sqrt{n_{1} n_{2}}}
$$

So that $S_{I}=S_{0}=D /(2-D)$ (assuming that $n_{1}=n_{2}$ ), and $S_{I}=S_{0}$ $=M R /(1-M R)$. For the case of two stimulus words $S_{I}\left(=S_{0}\right)$ is an increasing monotonic function of both $M R$ and $D$. Both $S_{1}$ $\left(=S_{0}\right)$ and $D$ range between 0 and 1 , while $M R$ ranges between 0 and .50 .

In the case of three stimulus words, the intersection coefficient is written as

$$
S_{1}=\frac{n_{123}}{\left(n_{1}+n_{2}+n_{3}\right)-\left(n_{12}+n_{13}+n_{23}\right)+n_{123}}
$$

while the overlap coefficient is written as

$$
S_{0}=\frac{n_{12}+n_{13}+n_{23}-2 n_{123}}{\left(n_{1}+n_{2}+n_{3}\right)-\left(n_{12}+n_{13}+n_{23}\right)+n_{123}}
$$

where $n_{1}, n_{2}$, and $n_{12}$ are defined as before and $n_{3}$ is the number of words in the set of responses $\left(A_{3}\right)$ to a third stimulus word; $n_{123}$ is the number of words which $A_{1}, A_{2}$, and $A_{3}$ have in common; $n_{13}$ is the number of words which $A_{1}$ and $A_{3}$ have in common; and $n_{23}$ is the number of words $A_{2}$ and $A_{3}$ have in common. In general, for $\mathbf{k}$ stimulus words, the intersection coefficient can be written as

$$
S_{I}=\frac{N_{1}^{k}}{\sum_{i=1}^{k}(-1)^{i+1} \sum_{j=1}^{i !(k-i) !} N_{j}^{i}}
$$

where $N_{i}=$ the number of words in the $j^{\text {th }} \mathrm{i}$-way intersection of Sets $\mathbf{A}_{1}, \mathbf{A}_{2}, \mathbf{A}_{3}, \ldots, \mathbf{A}_{k}$. Similarly, the overlap coefficient for $k$ stimulus words can be written as

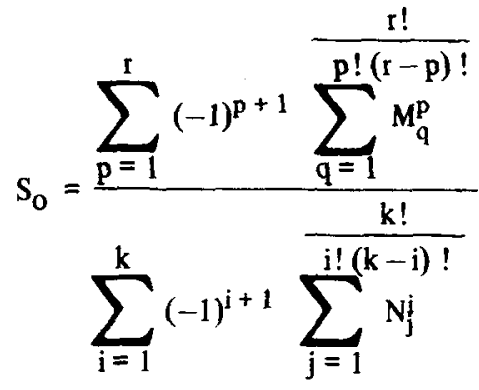

where $M_{q}^{p}$ is the number of elements in the $q$ th $p$-way intersection of Sets $\left(A_{1} \cap A_{2}\right),\left(A_{1} \cap A_{3}\right), \ldots,\left(A_{k-1} \cap A_{k}\right)$, and where $r=k ! /[2 !(k-2) !]$.

Both the index of total association (ITA) and index of concept cohesiveness (ICC) mentioned earlier are similar to the above coefficients. ITA is formulated as the ratio of the number of all responses in common to two or more stimulus words, to the total number of responses elicited by the stimulus words. ICC is the ratio of the number of responses in common to all of the stimuli being compared, to the number of responses in common to two or more of these stimulus words. Thus, ITA is analogous to $S_{0}$ and ICC is comparable to the ratio $S_{I} / S_{0}$.

The major difference between ITA, ICC, and the coefficients proposed here, $S_{I}$ and $S_{0}$, lies in the counting rule by which the numerator and denominator values are determined. For Marshall and Cofer (1963), the counting rule for determining commonality is the sum of all responses in common between stimulus words. On the other hand, we sum only the smallest number of common responses in the distributions being compared (this latter method is the same as the one used for both MR and D). In the case of the denominator of ITA, Marshall and Cofer simply sum all of the responses in all distributions, whereas we take the logical sum of these responses (counting each word only once). Another difference between our coefficients and ITA and ICC is that $S_{I}$ and $S_{0}$ assume the existence of representational responses (as in the computation of MR, MF, and D) while the computation of ITA and ICC does not.

Because the counting rules differ, no simple description of the relationship between coefficients such as was possible for MR and $\mathrm{D}$ is apparent. Consequently, we have prepared some illustrative data and computed ITA, ICC, $S_{1}$, and $S_{0}$ for the case of three stimulus words. These data and computations are presented in Table 1.

We have attempted to provide a single basis for formulating coefficients of relatedness that has the advantage of a single counting rule for any number of stimulus words, and that is 
Table 1

Computation of Coefficients of Relatedness for Three Stimulus Words (Entries are Frequencies)

\begin{tabular}{|c|c|c|c|}
\hline \multirow[b]{2}{*}{ Response } & \multicolumn{2}{|c|}{ Stimulus Word } & \multirow[b]{2}{*}{ Deer } \\
\hline & Pig & Horse & \\
\hline Pig & $(30)^{\mathrm{a}}$ & 0 & 1 \\
\hline Horse & 1 & $(30)$ & 2 \\
\hline Deer & 0 & 0 & $(30)$ \\
\hline Donkey & 15 & 0 & 0 \\
\hline Animal & 10 & 21 & 20 \\
\hline Cow & 4 & 4 & 4 \\
\hline Brown & 0 & 1 & 1 \\
\hline Man & 0 & 3 & 1 \\
\hline Run & 0 & 1 & 0 \\
\hline Ride & 0 & 0 & 1 \\
\hline \multicolumn{4}{|c|}{$\mathrm{ITA}=\frac{15+29+28}{90}=.800$} \\
\hline \multicolumn{4}{|c|}{$\mathrm{ICC}=\frac{14+25+24}{15+29+28}=.875$} \\
\hline \multicolumn{4}{|c|}{$S_{0}=\frac{(1+10+4)+(1+1+10+4)+(2+20+4+1+1)-2(1+10+4)}{(60+60+60)-(15+16+28)+15}=.213$} \\
\hline \multicolumn{4}{|c|}{$S_{I}=\frac{15}{136}=.110$} \\
\hline$\frac{\mathrm{S}_{\mathrm{I}}}{\mathrm{S}_{\mathrm{o}}}=.516$ & & & \\
\hline
\end{tabular}

a Representational responses were used in the calculation of $S_{I}$ and $S_{O}$ but not in the calculation of ITA and ICC. functionally related to a number of existing coefficients. While we have not developed our coefficients for the case of continued association, we regard the formulation presented here as applicable for such data when responses are weighted according to their order of occurrence.

Finally, it should be pointed out that no attempt has been made to study the sampling behavior of any of the coefficients presented. Rather, the goal has been to consider these coefficients solely as descriptive indices of associative relatedness.

\section{REFERENCES}

DEESE, J. On the structure of associative meaning. Psychological Review, $1962,69,169 \cdot 175$.

DEESE, J. The structure of associations in language and thought. Baltimore: Johns Hopkins Press, 1965.

GARSKOFF, B. E., \& HOUSTON, J. P. Measurement of verbal relatedness: An idiographic approach. Psychological Review, 1963, 70, 277-288.

MARSHALL, G. R., \& COFER, C. H. Associative indices as measures of word relatedness: A summary and comparison of ten methods. Journal of Verbal Learning \& Verbal Behavior, 1963, 1, 408-421.

\section{NOTES}

1. This work was supported in part by grants to the University of Minnesota Center for Research in Human Learning, from the National Science Foundation (GS-1761), the National Institute of Child Health and Human Development (HD-01136-04) and from the Graduate School of the University of Minnesota.

2. The anthors wish to ackrowledge the contribution to this paper of $P$. W. Fox, D. G. MacEachem, R. Priebe, and R. Sandborgh. 